\title{
Nodal domain and eigenvalue multiplicity of graphs
}

\author{
HaO Xu and Shing-Tung Yau
}

\begin{abstract}
We give a uniform proof of the upper and lower bounds of strong nodal domains for generalized Laplacians of discrete graphs. We also study the set of all possible numbers of strong nodal domains and the maximum multiplicity sequence of spectra.
\end{abstract}

AMS 2000 SUBJECT CLASSIFICATIONS: 05C50, 15A18.

KEYWORDS AND PHRASES: Nodal domain, eigenvalue multiplicity, Laplacian.

\section{Introduction}

Let $G=(V, E)$ be a graph with vertex set $V=\{1, \ldots, n\}$ and edge set $E$. Throughout the paper, a graph $G$ is undirected and simple (i.e., has no multi-edges or loops). We allow $G$ to be disconnected.

The Laplacian of $G$ is the matrix $L(G)=D-A$, where $D$ is the diagonal matrix whose entries are the degree of the vertices and $A$ is the adjacency matrix of $G$. Chung's normalized Laplacian $\tilde{L}(G)[6]$ is defined by

$$
\tilde{L}_{u v}= \begin{cases}1 & \text { if } u=v \text { and } d(u)>0 \\ -1 / \sqrt{d(u) d(v)} & \text { if } u v \in E \\ 0 & \text { otherwise }\end{cases}
$$

A symmetric $n \times n$ matrix $M$ is called a generalized Laplacian of $G$ if it has nonpositive off-diagonal entries and two distinct vertices $u$ and $v$ are adjacent if and only if $M_{u v}<0$. We denote by $\mathscr{M}(G)$ the set of all generalized Laplacians of $G$. Both $L(G)$ and $\tilde{L}(G)$ belong to $\mathscr{M}(G)$.

For a given $M \in \mathscr{M}(G)$, the eigenvalues of $M$ are enumerated by $\lambda_{1} \leq$ $\lambda_{2} \leq \cdots \leq \lambda_{n}$

The maximum multiplicity sequence of spectra of $G$ is denoted by $\delta(G)=$ $\left(\delta_{1}, \ldots, \delta_{n}\right)$, where $\delta_{k}$ is defined to be the largest number $r$ such that the $k$-th eigenvalue of some $M \in \mathscr{M}(G)$ has multiplicity $r$, namely

$$
\lambda_{1} \leq \cdots \leq \lambda_{k-1}<\lambda_{k}=\cdots=\lambda_{k+r-1}<\lambda_{k+r} \leq \cdots \leq \lambda_{n}
$$


Given an eigenfunction $f$ on $G$, recall that a strong nodal domain of $f$ is a maximal connected induced subgraph of $G$ such that $f$ is either strictly positive or strictly negative. Denote by $\mathfrak{S}(f)$ the number of strong nodal domains. As an analogue of Courant's nodal domain theorem for elliptic operators on manifolds, we have the following discrete nodal domain theorem of Davies et al. (cf. also [8]):

Theorem 1.1. [7] Let $M \in \mathscr{M}(G)$. Let $\lambda_{k}$ be the $k$-th eigenvalue with multiplicity $r$. Then for any eigenfunction $f$ corresponding to $\lambda_{k}$, we have $\mathfrak{S}(f) \leq k+r-1$.

Thus $\delta_{k}$ controls the upper bound of strong nodal domains.

Corollary 1.2. Let $M \in \mathscr{M}(G)$ and $f$ be an eigenfunction of its $k$-th eigenvalue. Then $\mathfrak{S}(f) \leq k+\delta_{k}-1$.

An upper bound of $\delta_{k}$ of 3-connected graphs in terms of the embedding genus into surfaces was obtained in [12]. Their proof used the method of Cheng [5] in estimating eigenvalue multiplicity of Riemann surfaces.

We have the following result on the lower bound of strong nodal domains.

Theorem 1.3. Let $M \in \mathscr{M}(G)$. Let $\lambda_{k}$ be the $k$-th eigenvalue with multiplicity $r$ and $f$ an eigenfunction corresponding to $\lambda_{k}$, which is zero on exactly $z$ vertices. Then $\mathfrak{S}(f) \geq k+r-1-\ell-z$, where $\ell$ is the minimal number of edges that need to be removed from $G$ in order to turn it into a forest.

We emphasize that the graph $G$ in Theorems 1.1 and 1.3 may be disconnected. Theorem 1.3 was first proved by Berkolaiko [1] under the stronger assumption that $G$ is connected, $\lambda_{k}$ is a simple eigenvalue and $f$ is not zero on any vertex.

The paper is organized as following: In Section 2, we give a uniform proof of Theorems 1.1 and 1.3. In Section 3, we study the gap phenomenon in the counting of strong nodal domains. In Section 4, we compute $\delta(G)$ for certain special graphs.

\section{Upper and lower bounds of strong nodal domains}

Given a graph $G=(V, E)$ (not necessarily connected). Labeling $V$ with $\{1, \ldots, n\}$, we may identify a function on $V$ as a vector $\left(x_{1}, \ldots, x_{n}\right)$. Let $c$ denote the number of connected components of $G$ and $\ell$ be the minimal number of edges that need to be removed from $G$ in order to turn it into a forest.

We need some standard results from linear algebra. 
Lemma 2.1. [9] Let $T$ be a tree with the set of vertices $\{1, \ldots, n\}$. Then the $n-1$ linear forms $x_{i}-x_{j}$ where $(i, j), i<j$, are edges of $T$, are linearly independent.

The following classical result is called the interlacing theorem.

Theorem 2.2. Let $A$ be a Hermitian matrix with eigenvalues $\lambda_{1}, \ldots, \lambda_{n}$ and $B$ be a principle submatrix with eigenvalues $\mu_{1}, \ldots, \mu_{m}$. Then we have the inequalities $\lambda_{i} \leq \mu_{i} \leq \lambda_{n-m+i}, \forall 1 \leq i \leq m$.

Lemma 2.3. Prescribe a sign $\{+,-\}$ at each vertex of $G$. Let $B=$ $\sum_{(i, j) \in E} a_{i j}\left(x_{i}-x_{j}\right)^{2}$ be a quadratic form satisfying $a_{i j}>0$ if $i$ and $j$ are of the same sign and $a_{i j}<0$ if $i$ and $j$ have different signs. We assume that the rank of $B$ equals $n-r$ and the positive index of inertia of $B$ equals $d$. Let $T$ be a spanning forest of the subgraph $H$ consisting of all edges $(i, j)$ such that $a_{i j}>0$. Then

$$
d \leq|E(T)| \leq|E(H)| \leq d+\ell .
$$

Similarly, let $T^{\prime}$ be a spanning forest of the subgraph $H^{\prime}$ consisting of all edges $(i, j)$ such that $a_{i j}<0$. Then

$$
n-r-d \leq\left|E\left(T^{\prime}\right)\right| \leq\left|E\left(H^{\prime}\right)\right| \leq n-r-d+\ell .
$$

Proof. By Sylvester's law of inertia, we can rewrite $B$ as

$$
B=\sum_{i=1}^{n-r} b_{i} Y_{i}^{2}
$$

where $Y_{i}=\sum_{j=1}^{n} m_{i j} x_{j}$ are independent linear forms and $b_{1}>0, \ldots, b_{d}>0$, $b_{d+1}<0, \ldots, b_{n-r}<0$. We shall prove (3) by contradiction.

First, we assume that $|E(T)|<d$, consider two systems of linear equations

$$
\left\{x_{i}-x_{j}=0\right\}_{(i, j) \in E(H)}, \quad Y_{d+1}=0, \ldots, Y_{n-r}=0
$$

and

$$
Y_{1}=0, \ldots, Y_{n-r}=0 .
$$

Since the rank of the system (7) is $n-r$ and by Lemma 2.1 the rank of the system (6) is $\leq|E(T)|+n-r-d<n-r$, there exists a nontrivial solution $\left(x_{1}^{0}, \ldots, x_{n}^{0}\right)$ of (6) which does not satisfy (7). It follows that 
$B\left(x_{1}^{0}, \ldots, x_{n}^{0}\right) \leq 0$ by $(2)$ and $B\left(x_{1}^{0}, \ldots, x_{n}^{0}\right)>0$ by $(3)$. We have reached a contradiction.

Next we assume $|E(H)|>d+\ell$. Consider two systems of linear equations

$$
Y_{1}=0, \ldots, Y_{d}=0, \quad\left\{x_{i}-x_{j}=0\right\}_{(i, j) \notin E(H)}
$$

and

$$
\left\{x_{i}-x_{j}=0\right\}_{(i, j) \in E(G)} .
$$

Since the rank of the system (9) is $n-c$ and the rank of the system (8) is $\leq d+n-c+\ell-|E(H)|<n-c$, there exists a nontrivial solution $\left(x_{1}^{0}, \ldots, x_{n}^{0}\right)$ of (8) which does not satisfy (9). It follows that $B\left(x_{1}^{0}, \ldots, x_{n}^{0}\right) \leq 0$ by (8) and $B\left(x_{1}^{0}, \ldots, x_{n}^{0}\right)>0$ by $(9)$. We again reached a contradiction. So we conclude the proof of (3). The proof of (4) is similar.

\subsection{Proof of Theorems 1.1 and 1.3}

The first half of the proof was adapted from the argument of Biyikoglu [2]. We first assume that $f$ does not vanish on any vertex. We divide $V(G)$ into three disjoint sets $P, S$ and $C$, where $P$ and $S$ denote the set of all vertices where $f$ is positive and negative respectively and which are incident to some edge where $f$ does not change sign. $C$ is the set of remaining vertices. Let $G[P]$ and $G[S]$ denote the induced subgraphs of $P$ and $S$ respectively. In fact, they consist of those edges where $f$ does not change sign. Let $p$ and $s$ be the number of components of $G[P]$ and $G(S)$. Let $T$ be a spanning forest of the disjoint union of $G[P]$ and $G[S]$. Then we have $|P|-p+|S|-s=$ $|E(T)|$.

Let $F$ be the a diagonal matrix with $F_{v v}=f(v), v \in V(G)$, and let $B=F\left(M-\lambda_{k} I\right) F$. Since $B$ satisfies $B_{u u}+\sum_{u v \in E(G)} B_{u v}=0, \forall u \in V(G)$.

We have $(B g)(x)=\sum_{u v \in E(G)}\left(-B_{u v}\right)(g(u)-g(v))$, from which we get

$$
\begin{aligned}
\langle g, B g\rangle & =\sum_{u v \in E(G)}\left(-B_{u v}\right)(g(u)-g(v))^{2} \\
& =\sum_{u v \in E(G)}\left(-M_{u v}\right) f(u) f(v)(g(u)-g(v))^{2}
\end{aligned}
$$

It follow from (3) that

$$
n-(k+r-1) \leq|E(T)| \leq n-(k+r-1)+\ell,
$$


which implies

$$
|P|+|S|-(n-(k+r-1))+\ell \leq p+s \leq|P|+|S|-(n-(k+r-1)) .
$$

By definition of $P, S$ and $C$, we have

$$
\mathfrak{S}(f)=|C|+p+s=n-(|P|+|S|)+p+s,
$$

together with (11), we get

$$
k+r-1-\ell \leq \mathfrak{S}(f) \leq k+r-1 .
$$

For general $f$ that is zero on some vertex, say $v$, then the restriction of $f$ to $G-v$ is still an eigenvector of $\lambda_{k}$, regarded as an eigenvalue of $G-v$. Assume that $\lambda_{k}$ is the $k^{\prime}$-th eigenvalue of $G-v$ with multiplicity $r^{\prime}$. By the interlacing theorem, it is not difficult to see that $k+r-1 \leq k^{\prime}+r^{\prime} \leq k+r$. So we get $\mathfrak{S}(f) \leq k^{\prime}+r^{\prime}-1 \leq k+r-1$ by induction on the number of vertices of $G$. If we assume that $f$ is zero on exactly $z$ vertices of $V(G)$, then by induction on $z$, we get $\mathfrak{S}(f) \geq k+r-1-\ell-z$. So we conclude the proof of Theorems 1.1 and 1.3 .

Corollary 2.4. Given a tree $T$ and $M \in \mathscr{M}(T)$, let $\lambda_{k}$ be the $k$-th eigenvalue with multiplicity $r$ and $f$ an eigenfunction corresponding to $\lambda_{k}$, which is zero on exactly $z$ vertices. Then $\mathfrak{S}(f) \geq k+r-1-z$.

Proof. It follows from Theorem 1.3, since $\ell=0$ for trees.

Corollary 2.5. Let $M \in \mathscr{M}(G)$ be a generalized Laplacian of a (not necessarily connected) graph $G$. If $f$ is an eigenfunction corresponding to an eigenvalue $\lambda$ which does not vanish on any vertex, then the multiplicity $r$ of $\lambda$ satisfies

$$
c \leq r \leq c+\ell
$$

Proof. Since $f$ is nonvanishing, $\lambda$ is an eigenvalue of $M$ restricted to each connected component. It follows that $r \geq c$.

Add up (3) and (4), we get

$$
|E(H)|+\left|E\left(H^{\prime}\right)\right| \leq n-r+2 \ell .
$$

Since $|E(H)|+\left|E\left(H^{\prime}\right)\right|=|E(G)|=n-c+\ell$, we get $r \leq c+\ell$. 
In Corollary 2.5, when $G$ is a tree, it is a well-known result of Fiedler [9] that $r=1$.

The sharpness of Theorem 1.3 and Corollary 2.5 can be seen as following:

Consider the Laplacian $L$ of the cycle graph $C_{n}, \lambda_{2}=2-2 \cos (2 \pi / n)$ has multiplicity 2 with an eigenfunction

$$
f=\left(\frac{\sqrt{2}}{2}, \sin \left(\frac{2 \pi}{n}+\frac{\pi}{4}\right), \ldots, \sin \left(\frac{2 \pi(n-1)}{n}+\frac{\pi}{4}\right)\right)
$$

which does not vanish on any vertex. We have $\mathfrak{S}(f)=2$.

Consider the Laplacian $L$ of the star graph $S_{n}, \lambda_{2}=1$ has multiplicity $n-2$ with an eigenfunction $f$ which is nonzero (taking 1 and -1 ) only on two degree one vertices. Then $\mathfrak{S}(f)=2$.

\section{Possible numbers of strong nodal domains}

If we prescribe a sign $\{+,-, 0\}$ on each vertex of $G$, denoting such a sign pattern by $s$, we can still define the number of strong nodal domains $\mathfrak{S}(s)$.

Lemma 3.1. Given a sign pattern $s$ on $G=(V, E)$, there is an eigenfunction $f$ of some $M \in \mathscr{M}(G)$ with the sign pattern $s$ if and only if for each vertex $v$ with $s(v)=0$, either all neighbors of $v$ are zero vertices or $v$ is adjacent to vertices with both + and - signs.

Proof. The necessity was proved in [3, p. 20]. Let $f$ be an eigenfunction of some $M \in \mathscr{M}(G)$, we may assume its eigenvalue equals 1 . If $f(z)=0$, then

$$
0=\sum_{x \in V} M_{z x} f(x)=\sum_{x \sim z} M_{z x} f(x)
$$

Since $M_{z x}<0$ when $x \sim z$, so we get the necessary condition if $s$ is the sign pattern corresponding to $f$.

Next we prove that if a sign pattern $s$ satisfies the given condition, then it must be the sign pattern of an eigenfunction $f$ with eigenvalue 0 . We may order the vertices so that $s=\left(s\left(v_{1}\right), \ldots, s\left(v_{p}\right), 0, \ldots, 0\right)^{T}$, where $s\left(v_{i}\right) \neq 0,1 \leq i \leq p$. Let $f\left(v_{i}\right)=s\left(v_{i}\right) \cdot 1, \forall 1 \leq i \leq n$. We want to construct a symmetric matrix $M \in \mathscr{M}(G)$ such that $M f=0$. Since $f\left(v_{i}\right) \neq 0,1 \leq i \leq p$ and the diagonals of $M \in \mathscr{M}(G)$ is unconstrained, we can arbitrarily specify $M_{i j}=M_{j i}$, where $(i, j) \in E, 1 \leq i<j \leq p$, such that

$$
M_{i i}=-\frac{1}{f\left(v_{i}\right)} \sum_{\substack{(i, j) \in E \\ 1 \leq j \leq p}} M_{i j} f\left(v_{j}\right), \quad 1 \leq i \leq p .
$$


Similarly it is not difficult to see that we can assign negative values to $M_{i j}$, where $p+1 \leq i \leq n, 1 \leq j \leq p,(i, j) \in E$, such that they satisfy the following system of linear equations

$$
\sum_{\substack{(i, j) \in E \\ 1 \leq j \leq p}} M_{i j} f\left(v_{j}\right)=0, \quad p+1 \leq i \leq n .
$$

Other entries of $M$ can be specified such that $M \in \mathscr{M}(G)$. Then $M$ constructed above must satisfy $M f=0$.

As pointed out in [3, p. 47], Lemma 3.1 belongs to the eigenvalue problem of sign-solvable linear systems studied in detail by Brualdi and Shader [4].

A sign pattern of $G$ satisfying the condition of Lemma 3.1 will be called an admissible sign pattern, its totality is denoted by $\mathscr{P}(G)$. The nodal domain count sequence of $G$ is the set $\eta(G)=\{\mathfrak{S}(s) \mid s \in \mathscr{P}(G)\}$ arranged in increasing order. Note a theorem of Oren [13] says that for a connected graph $G, \mathfrak{S}(s) \leq|V|-\chi+2$, where $\chi$ is the chromatic number of $G$. Moreover, $n \in \eta(G)$ if and only if $G$ is bipartite.

Example 3.2. Let $T$ be a tree with $n$ vertices. Then $\eta(T)=(1, \ldots, n)$. Actually we prove the stronger statement that for any $1 \leq k \leq n$, we can get $k$ strong nodal domains by assigning only $\{+,-\}$ signs. The proof is by induction on $n$. We take $x$ to be a leaf and consider the tree $T-\{x\}$. By assigning $\{+\}$ or $\{-\}$ to $x$, the rest of the proof is immediate.

The following example shows that $\eta(G)$ may have gaps, i.e. $k_{1}, k_{2} \in \eta(G)$ but $j \notin \eta(G)$ for some $j \in\left(k_{1}, k_{2}\right)$.

Example 3.3. Let $C_{n}$ be a cycle of length $n$. Then $\eta\left(C_{n}\right)=(1,2,4, \ldots$, $2[n / 2])$. We first prove that $\eta\left(C_{n}\right)$ must not contain any odd number greater than 1 . Obviously $\eta\left(C_{3}\right)=\{1,2\}$. The proof is by induction on $n$ and by contradiction. If $\mathfrak{S}(s)=2 a+1>1$ is an odd number for some admissible sign pattern $s$ of $C_{n}$, then either $s(u)=0$ for some vertex $u$ or there are two adjacent vertices $u$ and $v$ with $s(u)=s(v) \neq 0$. In the first case, the two neighboring vertices of $u$ must have different signs $\{+\}$ and $\{-\}$ respectively and we may remove $u$ and make the two neighbors of $u$ adjacent. In the second case, we may merge $u, v$ into a single vertex. Either of the two operations generates an admissible sign pattern on $C_{n-1}$ with $2 a+1$ strong nodal domains. We arrived at a contradiction by induction on $n$.

On the other hand, it is easy to see that for any even number $2 k \leq n$, we have $2 k \in \eta\left(C_{n}\right)$. 
The above two examples imply that for a connected unicycle graph $G$, other than the cycle, $\eta(G)$ has no gaps. The following example shows that the gaps in $\eta(G)$ may be arbitrarily large.

Example 3.4. Let $m \geq n \geq 1$ and $K_{m, n}=\left(V_{1}+V_{2}, E\right)$ be a complete bipartite graph with $\left|V_{1}\right|=m,\left|V_{2}\right|=n$ and every vertex in $V_{1}$ is adjacent to every vertex in $V_{2}$. Then $\eta\left(K_{m, n}\right)=(1, \ldots, m, m+n)$. For any $1 \leq k \leq m$, denote by $s$ the sign pattern that assigns $\{-\}$ to all vertices of $V_{2}$, assigns $\{+\}$ to $k-1$ vertices of $V_{1}$ and assigns $\{-\}$ to $n-k+1$ vertices of $V_{1}$. Then we have $\mathfrak{S}(s)=k$.

On the other hand, we have $k \notin \eta\left(K_{m, n}\right)$ for any $m<k<m+n$, since for a sign pattern $s$, if both $V_{1}$ and $V_{2}$ have signs $\{+\}$ and $\{-\}$, then $\mathfrak{S}(s)=2$.

The following lemma shows that for a bipartite graph $G$, a rather weak necessary condition for $\eta(G)$ to have no gaps is that $G$ must have a cut point.

Lemma 3.5. Let $G$ be a bipartite graph with $n \geq 3$ vertices. Then $n-1 \in$ $\eta(G)$ if and only if $G$ has a cut point.

Proof. If $p \in V$ is a cut point of $G$ and $G_{1}, \ldots, G_{k}, k \geq 2$ are the connected components of $G-\{p\}$, then we may define a sign pattern $s$ of $G$ by requiring that the restriction of $s$ to each $G_{i}$ has $\left|V_{i}\right|$ strong nodal domains. Finally set $s(p)=0$, by swapping the $\{+\}$ and $\{-\}$ signs on some $G_{i}$ if necessary, we get an admissible sign pattern $s$ and $\mathfrak{S}(s)=n-1$.

On the other hand, if $n-1 \in \eta(G)$, i.e. there exists an admissible sign pattern $s$ such that $\mathfrak{S}(s)=n-1$, then either there are two neighboring vertices $u, v$ having the same signs, say $s(u)=s(v)=\{+\}$ or there is a vertex $u$ such that $s(u)=0$. In the former case, $u, v$ could not lie on the same cycle, otherwise $\mathfrak{S}(s)<n-1$ since each cycle in $G$ has even length. Then at least one of $u, v$ is a cut point of $G$. In the latter case, $p$ must be adjacent to two vertices $u, v$ with $s(u)=\{+\}, s(v)=\{-\}$. Then $u, p, v$ could not lie on the same cycle since $G$ has only even cycles. So we proved that $p$ is a cut point of $G$.

It shall be interesting to find a characterization of those connected graphs $G$ such that $\eta(G)$ has no gaps.

\section{The maximum multiplicity sequence of spectra}

Without loss of generality, we may assume $G$ to be connected when talking about $\delta(G)$, the maximum multiplicity sequence of spectra defined in Section 1. 
Lemma 4.1. Given a connected graph $G$ with $\delta(G)=\left(\delta_{1}, \ldots, \delta_{n}\right)$, we have

i) $\delta_{1}=1$ and $1 \leq \delta_{k} \leq n+1-k, \forall 1 \leq k \leq n$.

ii) If $G$ is bipartite, then $\delta_{k} \leq n-k, \forall 1 \leq k \leq n$.

iii) If $G^{\prime}$ is obtained by removing a vertex from $G$ and $\delta\left(G^{\prime}\right)=\left(\delta_{1}^{\prime}, \ldots\right.$, $\left.\delta_{n-1}^{\prime}\right)$. Then $\delta_{k}-1 \leq \delta_{k}^{\prime} \leq \delta_{k+1}+1, \forall 1 \leq k \leq n-1$.

iv) $\delta_{k}=\max \left\{i_{0}(M) \mid M \in \mathscr{M}(G), i_{-}(M)=k-1\right\}$, where $\left(i_{+}(M), i_{-}(M)\right.$, $\left.i_{0}(M)\right)$ is the inertia of $M$.

v) $\max \left(\delta_{1}, \ldots, \delta_{n}\right)=n-\operatorname{mr}(G)$, where $\operatorname{mr}(G)=\min \{\operatorname{rank}(M) \mid M \in$ $\mathscr{M}(G)\}$.

vi) $\max \left(\delta_{1}, \ldots, \delta_{n}\right)=1$ if and only if $G=P_{n}$ the path.

vii) $\delta_{2}=n-1$ if and only if $G=K_{n}$ the complete graph.

Proof. $\delta_{1}=1$ follows from the Perron-Frobenius Theorem (cf. Corollary 2.23 in [3]). The inequality in (i) is obvious. (ii) follows from the fact that the largest eigenvalue of $M \in \mathscr{M}(G)$ is simple if $G$ is bipartite (cf. Corollary 3.25 in [3]). (iii) follows from the interlacing theorem (cf. Theorem 2.2). (iv) and (v) are obvious. (vi) and (vii) follow from (v) and respective characterizations of $P_{n}$ and $K_{n}$.

It would be interesting to compute or estimate these $\delta_{i}$ in terms of graph invariants. For trees, there is the following elegant result.

Theorem 4.2. [10] Let $T$ be a tree with $\delta(T)=\left(\delta_{1}, \ldots, \delta_{n}\right)$. Then

$$
\max \left(\delta_{1}, \ldots, \delta_{n}\right)=p(T),
$$

where $p(T)$ is the path cover number of $T$, i.e., the minimum number of vertex disjoint paths that cover all the vertices of $T$.

In fact, the authors of [10] considered more general real symmetric matrix $M$ such that two distinct vertices $u$ and $v$ in $T$ are adjacent if and only if $M_{u v} \neq 0$. However for such $M$, it is not difficult to see that there exists a nonsingular diagonal matrix $D$ such that the off-diagonal entries of $D M D$ is either 0 or -1 . Therefore, in the proof of Theorem 4.2, we may restrict to $M$ with $M_{u v}=-1$ for adjacent $u, v$.

In general, it is very difficult to compute $\delta(G)$ for an arbitrary graph $G$. We do not know an algorithm.

Example 4.3. We compute $\delta(G)$ for the five connected graphs with 4 vertices. By Lemma 4.1, it is easy to see that 


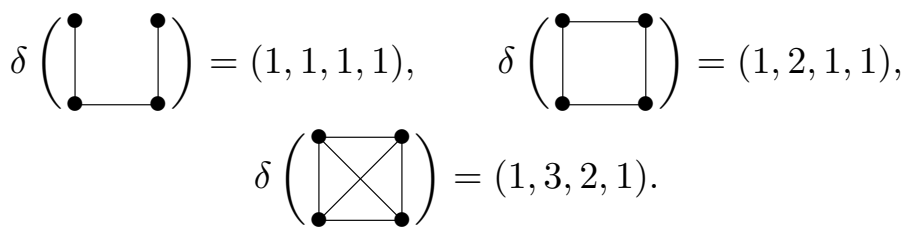

In order to show

$$
\delta\left(\prod_{\bullet}^{\bullet}\right)=(1,2,2,1) \text {, }
$$

we pick out two of its generalized Laplacians together with their characteristic polynomials:

$$
\begin{gathered}
\left.\begin{array}{cccc}
-1 & -1 & -1 & 0 \\
-1 & -1 & -1 & 0 \\
-1 & -1 & 0 & -1 \\
0 & 0 & -1 & 1
\end{array}\right] \\
\lambda^{2}\left(\lambda^{2}+\lambda-5\right)
\end{gathered} \quad\left[\begin{array}{cccc}
-1 & -1 & -1 & 0 \\
-1 & -1 & -1 & 0 \\
-1 & -1 & -2 & -1 \\
0 & 0 & -1 & -1
\end{array}\right]
$$

In order to show

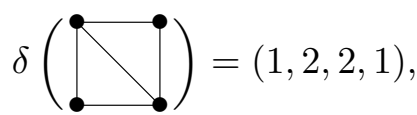

we pick out two of its generalized Laplacians together with their characteristic polynomials:

$$
\begin{array}{cccc}
{\left[\begin{array}{cccc}
0 & 0 & -1 & -1 \\
0 & 0 & -1 & -1 \\
-1 & -1 & -1 & -1 \\
-1 & -1 & -1 & -1
\end{array}\right]} & {\left[\begin{array}{cccc}
2 & 0 & -1 & -1 \\
0 & 2 & -1 & -1 \\
-1 & -1 & 3 & -1 \\
-1 & -1 & -1 & 3
\end{array}\right]} \\
\lambda^{2}\left(\lambda^{2}+2 \lambda-4\right) & \lambda^{2}\left(\lambda^{2}+5 \lambda+5\right)
\end{array}
$$

The remaining verifications are easy applications of Lemma 4.1.

Example 4.4. For the path $P_{n}$, the complete graph $K_{n}$ and the star $S_{n}$, we have

$$
\begin{aligned}
\delta\left(P_{n}\right) & =(1, \ldots, 1), \\
\delta\left(K_{n}\right) & =(1, n-1, n-2, \ldots, 1), \\
\delta\left(S_{n}\right) & =(1, n-2, n-3, \ldots, 2,1,1) .
\end{aligned}
$$


To prove (22), we consider the following generalized Laplacians of $K_{n}$ for $0 \leq k \leq n-2$,

$$
-J-\operatorname{diag}[\underbrace{1, \ldots, 1}_{k}, \underbrace{0, \ldots, 0}_{n-k}]
$$

where $J$ is the $n \times n$ matrix with all entries equal to 1 and $\operatorname{diag}[\cdots]$ denotes a diagonal matrix. Its characteristic polynomial $p(\lambda)$ equals

$$
p(\lambda)= \begin{cases}\lambda^{n-1}(\lambda+n), & \text { if } k=0, \\ \lambda^{n-1-k}(\lambda+1)^{k-1}\left(\lambda^{2}+(n+1) \lambda+n-k\right), & \text { if } 1 \leq k \leq n-2,\end{cases}
$$

which has no positive roots.

To prove (23), we just use (iii) of Lemma 4.1 and compute that for any $1 \leq k \leq n-2$, the characteristic polynomial of the following generalized Laplacians of $S_{n}$

$$
\left[\begin{array}{ccccccc}
0 & -1 & & & & & -1 \\
-1 & -1 & & & \\
& & \ddots & & \\
\vdots & & & -1 & \}_{0} & & \\
& & & & & \ddots & \\
-1 & & & & & & 0
\end{array}\right]_{n \times n}
$$

equals $\lambda^{n-2-k}(\lambda+1)^{k-1}\left(\lambda^{3}+\lambda^{2}-(n-1) \lambda-(n-1-k)\right)$, which has exactly one positive root.

Proposition 4.5. For the cycle graph $C_{n}, n \geq 3$, we have

$$
\delta\left(C_{n}\right)= \begin{cases}(1,2,1,2, \ldots, 1,2,1) & \text { if } n \text { is odd }, \\ (1,2,1,2, \ldots, 1,2,1,1) & \text { if } n \text { is even. }\end{cases}
$$

Proof. It is easy to see that $\operatorname{mr}\left(C_{n}\right)=n-2$. So $\delta_{i} \leq 2, \forall 1 \leq i \leq n$. Since the Laplace spectrum of $C_{n}$ is $2-2 \cos (2 \pi i / n), i=0, \ldots, n-1$, we have $\delta_{2 j}=2,1 \leq j \leq \frac{n-1}{2}$. So it remains to prove $\delta_{k}=1$ when $k$ is odd. Assume that $\delta_{k}=2$ for some $1 \leq k \leq n$. Without loss of generality, we may assume that the $k$-th eigenvalue $\lambda_{k}=0$, namely

$$
\lambda_{1} \leq \cdots \leq \lambda_{k-1}<\lambda_{k}=\lambda_{k+1}=0<\lambda_{k+2} \leq \cdots \leq \lambda_{n} .
$$


A generalized Laplacian $M \in \mathscr{M}\left(C_{n}\right)$ is of the form

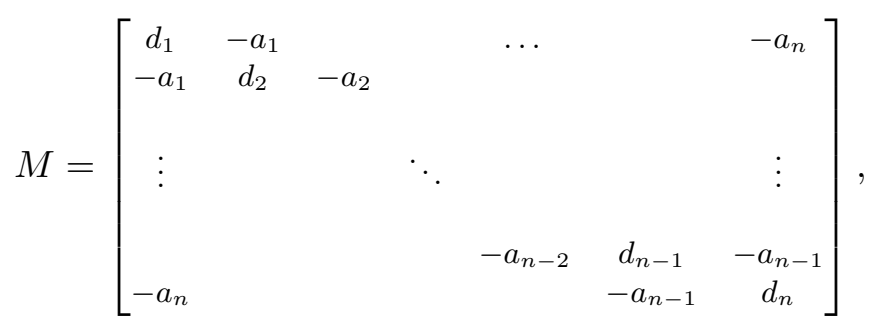

where $a_{i}>0,1 \leq i \leq n$. Assume $\operatorname{rank}(M)=n-2$, the characteristic polynomial of $M$ equals $\lambda^{2} p(\lambda)$ and

$$
p(0)=(-1)^{n} \sum_{1 \leq i<j \leq n} \operatorname{det}(M(\hat{i}, \hat{j})) \neq 0,
$$

where $M(\hat{i}, \hat{j})$ is the principle submatrix obtained by deleting the $i$-th $\& j$-th rows and columns. We shall prove that

$$
\operatorname{det}(M(\hat{i}, \hat{j})) \leq 0, \quad \forall 1 \leq i<j \leq n
$$

By symmetry, we may assume $i=1$. Since the matrix $M(\hat{1}, \hat{j})$ is of the form

$$
\left[\begin{array}{ll}
A & 0 \\
0 & B
\end{array}\right]
$$

where $A$ is a $(j-2) \times(j-2)$ matrix and $B$ is a $(n-j) \times(n-j)$ matrix. If we put back $i$-th row and $j$-th column, we get a $(n-1) \times(n-1)$ singular matrix $\tilde{M}$, since rank $M=n-2$. It is not difficult to check that

$$
\operatorname{det} \tilde{M}=(-1)^{j}\left(a_{1} \cdots a_{j-1} \cdot \operatorname{det} B+a_{j} \cdots a_{n} \cdot \operatorname{det} A\right)=0
$$

with the convention that $\operatorname{det} A=1$ if $j=2$; $\operatorname{det} B=1$ if $j=n$. So we get $\operatorname{det}(M(\hat{1}, \hat{j})) \leq 0$. Obviously (26) and (25) imply that

$$
\sum_{1 \leq i<j \leq n} \operatorname{det}(M(\hat{i}, \hat{j}))<0
$$

so by (25) there must be odd number of negative roots to the polynomial $p(\lambda)=0$, which implies that $k$ must be even. We conclude the proof.

The nonpositiveness of off-diagonal entries in the definition of generalized Laplacians is crucial for the validity of Proposition 4.5. For example, the 
following symmetric matrix (with underlying graph $C_{4}$ )

$$
\left[\begin{array}{cccc}
0 & -1 & 0 & -1 \\
-1 & 0 & -1 & 0 \\
0 & -1 & 0 & 1 \\
-1 & 0 & 1 & 0
\end{array}\right]
$$

has characteristic polynomial $\left(\lambda^{2}-2\right)^{2}$, so the third eigenvalue has multiplicity 2 .

Assume that $M \in \mathscr{M}(G)$ has distinct eigenvalues $\lambda_{1}<\lambda_{2}<\cdots<\lambda_{q}$ with multiplicity $m_{1}, \ldots, m_{q}$, then $\left(m_{1}, \ldots, m_{q}\right)$ is called the ordered multiplicity list of $M$.

Similar to Johnson et al. [11], one may ask the following problem: Given a connected graph $G$ and an ordered partition $\left(m_{1}, \ldots, m_{q}\right)$ of $n$, whether there exists some $M \in \mathscr{M}(G)$ such that $\left(m_{1}, \ldots, m_{q}\right)$ is the ordered multiplicity list of $M$. An obvious necessary condition is

$$
m_{j} \leq \delta_{b_{j}}, \quad \text { where } b_{j}=1+\sum_{i=1}^{j-1} m_{i}, \quad \forall 1 \leq j \leq q .
$$

From Example 4.3, it is not difficult to check that when $n \leq 4$, the above condition (27) is also sufficient.

\section{Acknowledgements}

We thank Yong Lin for helpful discussions.

\section{References}

[1] G. Berkolaiko, A lower bound for nodal count on discrete and metric graphs, Comm. Math. Phys. 278 (2008), 803-819. MR2373444

[2] T. Biyikoglu, A discrete nodal domain theorem for trees, Linear Algebra and Its Appl. 360 (2003), 197-205. MR1948481

[3] T. Biyikoglu, J. Leydold and P. F. Stadler, Laplacian Eigenvectors of Graphs: Perron-Frobenius and Faber-Krahn Type Theorems, Lecture Notes in Mathematics, vol. 1915, 2007. MR2340484

[4] R. A. Brualdi and B. L. Shader, Matrices of Sign-Solvable Linear Systems, Cambridge University Press, 1995. MR1358133 
[5] S. Y. Cheng, Eigenfunctions and nodal sets, Comment. Math. Helv. 51 (1976), 43-55. MR0397805

[6] F. R. K. Chung, Spectral Graph Theory, CBMS, vol. 92, AMS, Providence, RI, 1997. MR1421568

[7] E. B. Davies, G. M. L. Gladwell, J. Leydold, and P. F. Stadler, Discrete nodal domain theorems, Linear Algebra Appl. 336 (2001), 51-60. MR1855391

[8] A. M. Duval and V. Reiner, Perron-Frobenius type results and discrete versions of nodal domain theorems, Linear Algebra Appl. 294 (1999), 259-268. MR1693975

[9] M. Fiedler, Eigenvectors of acyclic matrices, Czech. Math. J. 25 (1975), 607-618. MR0387308

[10] C. R. Johnson and A. Leal Duarte, The maximum multiplicity of an eigenvalue in a matrix whose graph is a tree, Linear and Multilinear Algebra 46 (1999), 139-144. MR1712856

[11] C. R. Johnson and A. Leal Duarte, On the possible multiplicities of the eigenvalues of a Hermitian matrix whose graph is a tree, Linear Algebra Appl. 348 (2002), 7-21. MR1902112

[12] D. Mangoubi, Y. Lin, G. Lippner and S.-T. Yau, Nodal geometry of graphs on surfaces, Discrete Contin. Dyn. Syst. Ser. B 28(3) (2010), 1291-1298. MR2644790

[13] I. Oren, Nodal domain counts and the chromatic number of graphs, J. Phys. A: Math. Theor. 40 (2007), 9825-9832. MR2370546

HaO XU

Department of Mathematics

HARVARD UNIVERSITY

CAmbridge, MA 02138

USA

E-mail address: haoxu@math.harvard.edu

Shing-Tung YAu

Department of Mathematics

HARVARD UNIVERSITY

CAmbridge, MA 02138

USA

E-mail address: yau@math.harvard.edu

RECEIVED MARCH 4, 2012 